\title{
PDM creation and annihilation operators of the harmonic oscillators and the emergence of an alternative PDM-Hamiltonian
}

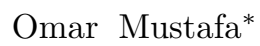 \\ Department of Physics, Eastern Mediterranean University, G. Magusa, north Cyprus, Mersin 10 - Turkey, \\ Tel.: +90 392 6301378; fax: +90 3692365 1604.
}

\begin{abstract}
The exact solvability and impressive pedagogical implementation of the harmonic oscillator's creation and annihilation operators make it a problem of great physical relevance and the most fundamental one in quantum mechanics. So would be the position-dependent mass (PDM) oscillator for the PDM quantum mechanics. We, hereby, construct the PDM creation and annihilation operators for the PDM oscillator via two different approaches. First, via von Roos PDM Hamiltonian and show that the commutation relation between the PDM creation $\hat{A}^{+}$and annihilation $\hat{A}$ operators, $\left[\hat{A}, \hat{A}^{+}\right]=1 \Leftrightarrow \hat{A} \hat{A}^{+}-1 / 2=\hat{A}^{+} \hat{A}+1 / 2$, not only offers a unique PDM-Hamiltonian $\hat{H}_{1}$ but also suggests a PDM-deformation in the coordinate system. Next, we use a PDM point canonical transformation of the textbook constant mass harmonic oscillator analog and obtain yet another set of PDM creation $\hat{B}^{+}$and annihilation $\hat{B}$ operators, hence an "apparently new" PDMHamiltonian $\hat{H}_{2}$ is obtained. The "new" PDM-Hamiltonian $\hat{H}_{2}$ turned out to be not only correlated with $\hat{H}_{1}$ but also represents an alternative and most simplistic user-friendly PDM-Hamiltonian, $\hat{H}=(\hat{p} / \sqrt{2 m(x)})^{2}+V(x) ; \hat{p}=-i \hbar \partial_{x}$, that has never been reported before.

Keywords: PDM harmonic oscillator, PDM creation and annihilation operators, von Roos PDM Hamiltonian, PDM point canonical transformation, alternative PDM-Hamiltonian.

PACS numbers: 05.45.-a, 03.50.Kk, 03.65.-w
\end{abstract}

\section{INTRODUCTION}

The harmonic oscillator problem is one of the most fundamental problems in classical and quantum mechanics. Its exact solvability and impressive/superb pedagogical implementation makes it a system of great physical relevance. Yet, its creation and annihilation operators play an important role in the build up of its energy basis that are vital components for perturbation theories treatments. The dynamics of a particle experiencing small fluctuations near the equilibrium point, $x_{\circ}$ say, allow us to express the corresponding potential as a Taylor series (i.e. perturbation series) expansion about $x_{\circ}$. On the other hand, particles with position-dependent mass (PDM) find their applicability in nuclear physics, nanophysics, semiconductor, etc [1-7]. In a more appropriate and instructive language, it would better be particles with position-dependent effective mass. That is, a deformation in the coordinate system may render the mass to be effectively position-dependent. A point mass moving within the curved coordinates/space transforms into position-dependent mass in Euclidean coordinates/space (c.f., e.g., 8 12] and references cited therein).. Such particles have been investigated for both classical and/or quantum systems over the years (e.g., see the sample of references $[1[35])$. It would be interesting, therefore, to put the PDM harmonic oscillator and its PDM creation and annihilation operators in their pedestal place so that they find their PDM exact solvability as well as PDM pedagogical implementation. This would be one of the most fundamental aspects of PDM quantum mechanics that inspires the content of the current methodical proposal.

In PDM quantum mechanics, one has to start with the most prominent von Roos PDM Hamiltonian [1]

$$
\hat{H}=-\frac{1}{4}\left[M(x)^{j} \partial_{x} M(x)^{k} \partial_{x} M(x)^{l}+M(x)^{l} \partial_{x} M(x)^{k} \partial_{x} M(x)^{j}\right]+V(x),
$$

and its ordering ambiguity conflict (an ambiguity that is manifested by the endless number of its kinetic energy operators that satisfy the von Roos constraint $j+k+l=-1$ ). That is, as one changes the values of the ordering ambiguity parameters $j, k$, and $l$ through the von Roos constraint, not only the profile of the kinetic energy operator will change but also the profile of the effective potential as well. On the theoretically and physically acceptable sides, nevertheless, it is found that the continuity conditions at the abrupt heterojunction suggest the parametric condition $j=l$ (c.f., e.g., [2, 4, 7, 27]). In the current methodical proposal we adopt this condition (i.e., $j=l$ ) and construct the PDM creation and annihilation operators. Two sets of such operators emerge in the process. The first of which

*Electronic address: omar.mustafa@emu.edu.tr 
emerges from the von Roos PDM Hamiltonian (1) and the other from a PDM point canonical transformation of the textbook constant mass analog of the harmonic oscillator. Each set of the PDM creation and annihilation operators results a PDM Hamiltonian that "looks" different from the other. They turn out to be correlated, nevertheless. We organize our paper in a sequential order, therefore..

In section 2, we recollect that under the classical PDM settings the force is no longer given by the time derivative of the linear momentum (i.e., $F \neq d(M(x) \dot{x}) / d t$ ) but it is rather given by $F=\sqrt{M(x)} d(\sqrt{M(x)} \dot{x}) / d t=-V^{\prime}(x)$ (c.f., e.g., [18] for more details on this issue). Consequently, we argue that the interaction potential energy $V(x)$ will be deformed to accommodate the new PDM force settings. That is, the traditional constant mass harmonic oscillator potential $V(x)=m_{\circ} \omega^{2} x^{2} / 2$ is deformed into a PDM harmonic oscillator potential $V(x)=m_{\circ} \omega^{2} Q(x) x^{2} / 2$, where $Q(x)$ is a PDM manifested deformation function to be strictly determined in the process of identifying the PDM creation and annihilation operators. In the first part of the same section, we use the von Roos PDM Hamiltonian along with a factorizing recipe to construct PDM creation and annihilation operators, $\hat{A}^{+}$and $\hat{A}$, respectively. Such PDM operators satisfy the commutation relation $\left[\hat{A}, \hat{A}^{+}\right]=1$ which consequently implies that $\hat{A} \hat{A}^{+}-1 / 2=\hat{A}^{+} \hat{A}+1 / 2$. The latter is not only used to build the harmonic oscillator PDM Hamiltonian $\hat{H}_{1}=\omega\left(\hat{A} \hat{A}^{+}-\frac{1}{2}\right)=\omega\left(\hat{A}^{+} \hat{A}+\frac{1}{2}\right)$ as usual, but also we use it to single out one unique PDM kinetic energy operator. In the second part of the same section, we use a PDM point canonical transformation of the constant mass textbook analog of the harmonic oscillator and build up yet an alternative harmonic oscillator PDM Hamiltonian $\hat{H}_{2}=\omega\left(\hat{B} \hat{B}^{+}-\frac{1}{2}\right)=\omega\left(\hat{B}^{+} \hat{B}+\frac{1}{2}\right)$ where $\hat{B}^{+}$ and $\hat{B}$ are alternative PDM creation and annihilation operators, satisfying the commutation relation $\left[\hat{B}, \hat{B}^{+}\right]=1$. The two "apparently different" PDM Hamiltonians $\hat{H}_{1}$ and $\hat{H}_{2}$ turned out (in the third part of section 2) to be correlated in such a way that they are alternative forms of each other. A sample of illustrative examples are given in section 3. Where we use the PDM-deformation $Q(x)$ to find the corresponding $m(x)$, and the PDM-deformed harmonic oscillator potential (first three examples). Next, we use the PDM $m(x)$ to obtain the corresponding PDMdeformation $Q(x)$ and the PDM-deformed harmonic oscillator potential (4th and 5th examples). Finally, we use a Morse-type PDM-deformed harmonic oscillator potential (6th example) and a Yukawa-type PDM-deformed harmonic oscillator potential (7th example) to find the corresponding PDM-deformations $Q(x)$ as well as the corresponding PDM functions $m(x)$. In our concluding remarks, section 4, we analyze our results and suggest a new alternative PDM Hamiltonian (in (70) below) as the most simplistic and user-friendly ever been reported in the literature. To the best of our knowledge, such results have never been reported elsewhere.

\section{PDM CREATION AND ANNIHILATION HARMONIC OSCILLATOR OPERATORS}

In an earlier work. Mustafa [18] has asserted that the force under PDM settings is no longer given by the time derivative of the linear momentum (i.e., $F \neq d p(x) / d t ; p(x)=M(x) \dot{x}, M(x)=m_{\circ} m(x)$ ) but it is rather given by

$$
F=\sqrt{m(x)} \frac{d}{d t}\left(m_{\circ} \sqrt{m(x)} \dot{x}\right)=m_{\circ} m(x) \ddot{x}+\frac{1}{2} m_{\circ} m^{\prime}(x) \dot{x}^{2}=-V^{\prime}(x) ; V^{\prime}(x)=\frac{d V(x)}{d x}, \dot{x}=\frac{d x}{d t} .
$$

For quasi-free PDM particles (i.e., $V(x)=0$ ), for example, it implies that while the PDM pseudo-momentum $\pi(x)=m_{\circ} \sqrt{m(x)} \dot{x}$ is a conserved quantity, the PDM linear momentum is no more a conserved quantity. It is, therefore, natural and convenient to assume that under PDM-settings the harmonic oscillator potential is deformed in such a way that the constant mass harmonic oscillator potential $m_{\circ} \omega^{2} x^{2} / 2$ transforms into

$$
V(x)=\frac{1}{2} m_{\circ} \omega^{2} Q(x) x^{2}=\frac{1}{2} m_{\circ} \omega^{2} q(x)^{2} ; q(x)=\sqrt{Q(x)} x .
$$

As long as $q(x)$ (consequently the PDM manifested deformation $Q(x)$ ) is to be determined in the process of constructing the PDM creation $\hat{A}^{+}$and annihilation $\hat{A}$ operators, this assumption remains sufficient and valid. Moreover, the PDM creation and annihilation operators should satisfy the commutation relation $\left[\hat{A}, \hat{A}^{+}\right]=1$ as is the case for constant mass settings. Keeping all this in mind, we construct such PDM operators in two different ways, the first of which is via the PDM von Roos Hamiltonian (1) and the second is via a PDM point canonical transformation of their constant mass textbook analog. Two apparently different PDM Hamiltonians emerge and the correlation between them is nevertheless identified. 


\section{A. via PDM von Roos Hamiltonian}

Enforcing the continuity conditions at the abrupt heterojunction necessarily implies $j=l$ in (1). Yet, the von Roos constraint, $j+k+l=-1$, for $j=l=a$ and $k=2 b$ would allow Hamiltonian (1) to collapse into a PDM Hamiltonian (with $m_{\circ}=\hbar=c=1$ units and $M(x)=m_{\circ} m(x)$ )

$$
\hat{H}_{1}=-\frac{1}{2} m(x)^{a} \partial_{x} m(x)^{2 b} \partial_{x} m(x)^{a}+\frac{1}{2} \omega^{2} q(x)^{2} ; a+b=-\frac{1}{2}, q(x)=\sqrt{Q(x)} x
$$

where $V(q(x))=\frac{1}{2} \omega^{2} q(x)^{2}$ identifies the PDM-deformed oscillator potential and $q(x)$ is a position-dependent mass function to be determined in the sequel (hence, $Q(x)$ will be determined). However, let us begin with the construction of the PDM harmonic oscillator creation and annihilation operators. In so doing, we may appeal to a factorizing recipe and temporarily suggest that the PDM harmonic oscillator creation operator $\hat{A}^{+}$is given by

$$
\hat{A}^{+}=-\frac{1}{\sqrt{2 \omega}} m(x)^{a} \partial_{x} m(x)^{b}+\sqrt{\frac{\omega}{2}} q(x),
$$

and the annihilation operator $\hat{A}$ by

$$
\hat{A}=\frac{1}{\sqrt{2 \omega}} m(x)^{b} \partial_{x} m(x)^{a}+\sqrt{\frac{\omega}{2}} q(x),
$$

The harmonic oscillator operators, however, are known to satisfy the commutation relation

$$
\left[\hat{A}, \hat{A}^{+}\right]=1 \Longleftrightarrow \hat{A}^{+} \hat{A}+\frac{1}{2}=\hat{A} \hat{A}^{+}-\frac{1}{2} .
$$

This commutation relation is a necessary and sufficient condition on the traditional constant mass harmonic oscillator creation and annihilation operators. So should be the case for the PDM harmonic oscillator creation and annihilation operators.

Under such settings and in a straightforward manner, one finds that

$$
\hat{A}^{+} \hat{A}=-\frac{1}{2 \omega} m(x)^{a} \partial_{x} m(x)^{2 b} \partial_{x} m(x)^{a}-2 a q(x)\left(\frac{1}{\sqrt{m(x)}}\right)^{\prime}-\left(\frac{q(x)}{2 \sqrt{m(x)}}\right)^{\prime}+\frac{\omega}{2} q(x)^{2}
$$

and

$$
\hat{A} \hat{A}^{+}=-\frac{1}{2 \omega} m(x)^{b} \partial_{x} m(x)^{2 a} \partial_{x} m(x)^{b}-2 a q(x)\left(\frac{1}{\sqrt{m(x)}}\right)^{\prime}-\left(\frac{q(x)}{2 \sqrt{m(x)}}\right)^{\prime}+\frac{\omega}{2} q(x)^{2}+\frac{q^{\prime}(x)}{\sqrt{m(x)}}
$$

The substitution of (8) and (9) in (7) would imply

$$
-\frac{1}{2} m(x)^{a} \partial_{x} m(x)^{2 b} \partial_{x} m(x)^{a}=-\frac{1}{2} m(x)^{b} \partial_{x} m(x)^{2 a} \partial_{x} m(x)^{b}+\frac{q^{\prime}(x)}{\sqrt{m(x)}}-1 .
$$

This result clearly suggests that the potential related terms vanish to yield

$$
\frac{q^{\prime}(x)}{\sqrt{m(x)}}-1=0 \Longleftrightarrow q(x)=\int \sqrt{m(x)} d x=\sqrt{Q(x)} x
$$

and the kinetic energy terms are equal, i.e.,

$$
-\frac{1}{2} m(x)^{a} \partial_{x} m(x)^{2 b} \partial_{x} m(x)^{a}=-\frac{1}{2} m(x)^{b} \partial_{x} m(x)^{2 a} \partial_{x} m(x)^{b} \Longleftrightarrow a=b .
$$

Hence, two basic and critical results are obtained here. The first of which identifies the form of $q(x)$ in $(11)$ (hence, relates $Q(x)$ with $m(x))$ and the second restricts the ambiguity parameters to the identity $a=b$ in (12). Yet, the substitution of $a=b$ into the von Roos constraint $a+b=-1 / 2$ would result in $a=b=-1 / 4$. Consequently, the PDM harmonic oscillator creation (5) and annihilation (6) operators would, respectively, read

$$
\hat{A}^{+}=-\frac{1}{\sqrt{2 \omega}} \frac{1}{\sqrt[4]{m(x)}} \partial_{x} \frac{1}{\sqrt[4]{m(x)}}+\sqrt{\frac{\omega}{2}} q(x) \Longleftrightarrow \hat{A}^{+}=-i\left(\frac{\hat{p}(x)}{\sqrt{2 \omega m(x)}}\right)+\sqrt{\frac{\omega}{2}} q(x),
$$


and

$$
\hat{A}=\frac{1}{\sqrt{2 \omega}} \frac{1}{\sqrt[4]{m(x)}} \partial_{x} \frac{1}{\sqrt[4]{m(x)}}+\sqrt{\frac{\omega}{2}} q(x) \Longleftrightarrow \hat{A}(x)=i\left(\frac{\hat{p}(x)}{\sqrt{2 \omega m(x)}}\right)+\sqrt{\frac{\omega}{2}} q(x),
$$

where

$$
\hat{p}(x)=-i\left(\partial_{x}-\frac{1}{4} \frac{m^{\prime}(x)}{m(x)}\right)
$$

is the PDM-momentum operator that has been very recently constructed and reported by Mustafa and Algadhi [10]. At this point, one may immediately show that the PDM Hamiltonian $\hat{H}_{1}$ of (4) satisfies the textbook relation

$$
\hat{H}_{1}=\omega\left(\hat{A} \hat{A}^{+}-\frac{1}{2}\right)=\omega\left(\hat{A}^{+} \hat{A}+\frac{1}{2}\right)
$$

and admits its differential form as

$$
\hat{H}_{1}=-\frac{1}{2} \frac{1}{\sqrt[4]{m(x)}} \partial_{x} \frac{1}{\sqrt{m(x)}} \partial_{x} \frac{1}{\sqrt[4]{m(x)}}+\frac{\omega^{2}}{2} q(x)^{2}
$$

As such, the so called ambiguity parameters in (4) are no longer ambiguous but are strictly determined to yield one unique representation for the PDM kinetic energy operator as

$$
\hat{T}_{1}=-\frac{1}{2} \frac{1}{\sqrt[4]{m(x)}} \partial_{x} \frac{1}{\sqrt{m(x)}} \partial_{x} \frac{1}{\sqrt[4]{m(x)}}=\left(\frac{\hat{p}(x)}{\sqrt{2 m(x)}}\right)^{2} .
$$

Which, in fact, imitates the kinetic energy operator $\hat{T}=\left(\hat{p} / \sqrt{2 m_{\circ}}\right)^{2}$ for constant mass settings. At this point, we may recollect that such parametric ordering in $\hat{T}_{1}$ of (18) is known in the literature as Mustafa and Mazharimousavi's ordering [27] (who have used a simple factorization approach for the von Roos PDM Hamiltonian (1) in general and found that $j=l=-1 / 4$ and $k=-1 / 2$ ). Yet, Cruz et al. [21] have used a supersymmetric approach and geometrically, shape-wise, compared the corresponding effective potentials (in terms of superpotentials) with the classical oscillator one ( using different values for $a$ ) and found that this PDM kinetic energy operator is graphically and asymptotically the most suitable ordering.

As a result, we may rewrite the PDM harmonic oscillator Hamiltonian in its most user-friendly form as

$$
\hat{H}_{1}=\left(\frac{\hat{p}(x)}{\sqrt{2 m(x)}}\right)^{2}+\frac{\omega^{2}}{2} q(x)^{2} ; \quad q(x)=\sqrt{Q(x)} x=\int \sqrt{m(x)} d x .
$$

One should notice that the PDM harmonic oscillator creation $\hat{A}^{+}$and annihilation $\hat{A}$ operators given in terms of the PDM-momentum operator in (13) and (14) clearly inherit the textbook forms for constant mass settings, where $m(x) \longrightarrow m_{\circ}$ and $\hat{p}(x) \longrightarrow \hat{p}=-i \partial_{x}$. Yet, the commutation relations for constant mass settings are also satisfied by the PDM settings. That is, one may easily show that

$$
[x, \hat{p}(x)]=i,\left[\hat{A}, \hat{H}_{1}\right]=\hat{A},\left[\hat{A}^{+}, \hat{H}_{1}\right]=-\hat{A}^{+},\left[\hat{A}^{+} \hat{A}, \hat{A}\right]=-\hat{A},\left[\hat{A}^{+} \hat{A}, \hat{A}^{+}\right]=\hat{A}^{+},
$$

\section{B. via a PDM point canonical transformation of the constant mass analog}

Here, we consider a particle with constant mass $m_{\circ}$ moving in the generalized coordinate $q$ and experiencing a textbook constant mass harmonic oscillator potential $V(q)=\frac{1}{2} m_{\circ} \omega^{2} q^{2}$. The Hamiltonian describing this problem reads (with $m_{\circ}=\hbar=c=1$ units )

$$
\hat{H}_{2}=-\frac{1}{2} \partial_{q}^{2}+\frac{1}{2} \omega^{2} q^{2}
$$

We may now recollect that the corresponding textbook creation and annihilation operators are, respectively, given by 


$$
\hat{B}^{+}=-\frac{1}{\sqrt{2 \omega}} \partial_{q}+\sqrt{\frac{\omega}{2}} q
$$

and

$$
\hat{B}=\frac{1}{\sqrt{2 \omega}} \partial_{q}+\sqrt{\frac{\omega}{2}} q
$$

where

$$
\hat{H}_{2}=\omega\left(\hat{B} \hat{B}^{+}-\frac{1}{2}\right)=\omega\left(\hat{B}^{+} \hat{B}+\frac{1}{2}\right) \Longleftrightarrow\left[\hat{B}, \hat{B}^{+}\right]=1 .
$$

Next, let us use a PDM point canonical transformation in the form of

$$
q=q(x)=\int \sqrt{m(x)} d x \Longleftrightarrow d q=\sqrt{m(x)} d x \Longleftrightarrow \partial_{q}=\frac{1}{\sqrt{m(x)}} \partial_{x},
$$

similar to (11). This would necessarily transform the constant mass creation (22) and annihilation (23) operators into PDM creation and annihilation operators which are, respectively,

$$
\hat{B}^{+}=-\frac{1}{\sqrt{2 \omega m(x)}} \partial_{x}+\sqrt{\frac{\omega}{2}} q(x)
$$

and

$$
\hat{B}=\frac{1}{\sqrt{2 \omega m(x)}} \partial_{x}+\sqrt{\frac{\omega}{2}} q(x) .
$$

Which would, in turn, allow us to write the PDM form of $\hat{H}_{2}$ in (24) as

$$
\hat{H}_{2}=-\frac{1}{2} \frac{1}{\sqrt{m(x)}} \partial_{x} \frac{1}{\sqrt{m(x)}} \partial_{x}+\frac{\omega^{2}}{2} q(x)^{2}, q(x)=\sqrt{Q(x)} x .
$$

It is obvious and crystal clear that the PDM Hamiltonian $\hat{H}_{1}$ of (17) (i.e., a von Roos PDM Hamiltonian descendent) and the PDM Hamiltonian $\hat{H}_{2}$ of (28) (i.e., a PDM point canonical transformation descendent of the constant mass textbook analog) are apparently not the same. However, it is still premature to jump to conclusions at this point, for one has to check their corresponding eigenvalues and eigenfunctions and perhaps find out a correlation between them. This is done in the sequel.

\section{Correlation between the two PDM Hamiltonians $\hat{H}_{1}$ and $\hat{H}_{2}$}

Let us assume that the PDM Hamiltonian $\hat{H}_{1}$ of $(17)$ operates on a wavefunction $\phi(x)$ so that the corresponding PDM Schrödinger equation reads

$$
\hat{H}_{1} \phi(x)=E_{1} \phi(x) \Longleftrightarrow\left\{-\frac{1}{2} \frac{1}{\sqrt[4]{m(x)}} \partial_{x} \frac{1}{\sqrt{m(x)}} \partial_{x} \frac{1}{\sqrt[4]{m(x)}}+\frac{\omega^{2}}{2} q(x)^{2}\right\} \phi(x)=E_{1} \phi(x) .
$$

On the other hand, the PDM Hamiltonian $\hat{H}_{2}$ of $(28)$ is assumed to operate on a wavefunction $\Psi(q)=\Psi(q(x))$ so that the corresponding PDM Schrödinger equation reads

$$
\hat{H}_{2} \Psi(q(x))=E_{2} \Psi(q(x)) \Longleftrightarrow\left\{-\frac{1}{2} \frac{1}{\sqrt{m(x)}} \partial_{x} \frac{1}{\sqrt{m(x)}} \partial_{x}+\frac{\omega^{2}}{2} q(x)^{2}\right\} \Psi(q(x))=E_{2} \Psi(q(x)) .
$$

Our objective here is to bring the PDM Schrödinger equation (29) into a similar form as that of (30). In so doing, let us multiply $(29)$, from the left, by $1 / \sqrt[4]{m(x)}$ and rearrange terms to get

$$
\left\{-\frac{1}{2} \frac{1}{\sqrt{m(x)}} \partial_{x} \frac{1}{\sqrt{m(x)}} \partial_{x}+\frac{\omega^{2}}{2} q(x)^{2}\right\} m(x)^{-1 / 4} \phi(x)=E_{1} m(x)^{-1 / 4} \phi(x) .
$$


Therefore, if one demands that the PDM Hamiltonian $\hat{H}_{1}$ of (17) is isospectral with the PDM Hamiltonian $\hat{H}_{2}$ of $(28)$ (which is indeed the case here, for we have the very same PDM harmonic oscillator problem for both Hamiltonians) we may then conclude that

$$
\Psi(q(x))=m(x)^{-1 / 4} \phi(x) \Longleftrightarrow E_{1}=E_{2}=E .
$$

Consequently, we may now safely argue that

$$
\hat{H}_{2} \Psi(q(x))=m(x)^{-1 / 4} \hat{H}_{1} \phi(x)=E \Psi(q(x))=E m(x)^{-1 / 4} \phi(x) .
$$

Then, the correlation between the two sets of PDM creation and annihilation operators now reads

$$
\left(\hat{A} \hat{A}^{+}-\frac{1}{2}\right) \phi(x)=m(x)^{1 / 4}\left(\hat{B} \hat{B}^{+}-\frac{1}{2}\right) \Psi(q(x)) .
$$

The connection and mapping between the two PDM systems is made clear, therefore.

At this point, however, the reader should be aware of the fact that the eigenvalues $E$ and eigenfunctions $\Psi(q(x))$ are nothings but the exact textbook harmonic oscillator's and are, respectively, given by

$$
E_{n}=\omega\left(n+\frac{1}{2}\right), \Psi_{n}(q(x))=\mathcal{N}_{n} \exp \left(-\frac{q(x)^{2}}{2}\right) H_{n}(q(x)) ; n=0,1,2, \cdots,
$$

where

$$
H_{n}(q(x))=H_{n}(q)=(-1)^{n} \exp \left(q^{2}\right) \frac{d^{n}}{d q^{n}} \exp \left(-q^{2}\right)
$$

are the Hermit polynomials and $n$ represents the principle quantum number. Yet, both $\hat{H}_{2} \Psi(q(x))$ and $\hat{H}_{1} \phi(x)$ would result a textbook like PDM Schrödinger equation in the form

$$
\left\{\left(\frac{\hat{p}(x)}{\sqrt{2 m(x)}}\right)^{2}+\frac{\omega^{2}}{2} q(x)^{2}\right\} \phi_{n}(x)=E_{n} \phi_{n}(x),
$$

which, indeed, not only replicates the traditional Schrödinger equation but also collapses exactly into that for constant mass settings. Moreover, the eigenvalues and eigenfunctions are readily exactly known for all integrable $\sqrt{m(x)}$ of $q(x)$ in (11). A sample of illustrative examples is given below.

\section{A SAMPLE OF ILLUSTRATIVE EXAMPLES}

Apriori, wee have asserted that $q(x)$ of (11) determines the relation between $Q(x)$ and $m(x)$. That is, while the second part of (11) yields that

$$
q(x)=\sqrt{Q(x)} x \Longleftrightarrow q^{\prime}(x)=\frac{d q(x)}{d x}=\sqrt{Q(x)}\left(1+\frac{Q^{\prime}(x)}{2 Q(x)} x\right),
$$

the first part of (11), on the other hand, implies (compared with (38)) that

$$
q^{\prime}(x)=\sqrt{m(x)} \Longleftrightarrow \sqrt{m(x)}=\sqrt{Q(x)}\left(1+\frac{Q^{\prime}(x)}{2 Q(x)} x\right) .
$$

This result would determine the form of $q(x)$ in (35) and consequently the form of the PDM harmonic oscillator potential

$$
V(x)=\frac{\omega^{2}}{2} Q(x) x^{2}
$$

where $Q(x)$ is a dimensionless scalar multiplier that represents, hereinafter, a PDM-deformation function (introduced as a manifestation of the PDM setting) of the constant mass harmonic oscillator potential $\omega^{2} x^{2} / 2$. Therefore, our potential $V(x)$ in (40) represents a PDM-deformed harmonic oscillator potential. Yet at this point, it should be 
noted that $Q(x)=$ const. and $Q(x)=m(x)$ would immediately retrieve the constant mass setting, and shall not be discussed here, therefore. Under such PDM implications, the PDM harmonic oscillator potential can never be expressed as $V(x)=m(x) \omega^{2} x^{2} / 2$ but rather it should be expressed as in (40) where condition (39) determines the nature of the relation between PDM-deformation function $Q(x)$ and $m(x)$ (c.f., e.g., Cruz cruz et al. [21] and Carinena et al. 34]).

Apart from the constant mass setting, we now consider the following sample of illustrative examples. All of which admit exact eigenvalues and eigenfunctions inherited from our results in (33), (35), and (36) as the exact solutions for the PDM Schrödinger equation in (37). They are given, respectively, as

$$
E_{n}=\omega\left(n+\frac{1}{2}\right), \phi_{n}(x)=m(x)^{1 / 4} \Psi_{n}(q(x)), \Psi_{n}(q(x))=\mathcal{N}_{n} \exp \left(-\frac{q(x)^{2}}{2}\right) H_{n}(q(x)) ; n=0,1,2, \cdots,
$$

provided that $Q(x)$ (or equivalently $m(x)$ ), is determined through (39). This is not only restricted to the sample of illustrative examples below but also for every $Q(x)$ and $m(x)$ satisfying (39) provided that they are, mathematical and/or quantum mechanical wise, well-behaved functions (see, for example, the sample of PDM-functions in [21, 34], some of which are used below).

\section{A. A PDM sample case without singularities $m(x)=\left(1+\lambda x^{2}\right)^{-3}$}

The assumption that the PDM deformation function $Q(x)$ is given by

$$
Q(x)=\frac{1}{1+\lambda x^{2}}
$$

without singularities, would allow us to obtain, through (39), a PDM without singularities as

$$
m(x)=\frac{1}{\left(1+\lambda x^{2}\right)^{3}} \Longleftrightarrow q(x)=\frac{x}{\sqrt{1+\lambda x^{2}}} .
$$

Consequently, the PDM-deformed harmonic oscillator potential (40) reads

$$
V(x)=\frac{\omega^{2}}{2}\left(\frac{x^{2}}{1+\lambda x^{2}}\right),
$$

to admit the exact eigenvalues and eigenfunctions in (41) as the solutions of the PDM-Schrödinger equation (37) with $q(x)$ of (43).

\section{B. A PDM sample case with singularities $m(x)=\left(\lambda x^{2}-1\right)^{-3}$}

If we consider that the PDM-deformation function $Q(x)$ has singularities and given by

$$
Q(x)=\frac{1}{\lambda x^{2}-1},
$$

then a PDM with singularities is obtained as

$$
m(x)=\frac{1}{\left(\lambda x^{2}-1\right)^{3}} \Longleftrightarrow q(x)=\frac{x}{\sqrt{\lambda x^{2}-1}}
$$

and the corresponding PDM-deformed harmonic oscillator potential (40) reads

$$
V(x)=\frac{\omega^{2}}{2}\left(\frac{x^{2}}{\lambda x^{2}-1}\right) .
$$

with the exact eigenvalues and eigenfunctions in (41) as the solutions of the PDM-Schrödinger equation (37) with $q(x)$ of (43). 


\section{A power-law PDM sample case $m(x) \sim x^{\sigma}$}

A power-law PDM-deformation function

$$
Q(x)=\lambda x^{\sigma},
$$

would lead to a PDM function

$$
m(x)=\left(1+\frac{\sigma}{2}\right)^{2} \lambda x^{\sigma} \Longleftrightarrow q(x)=\sqrt{\lambda} x^{(\sigma+2) / 2},
$$

where $\sigma \neq-2,0 ; \sigma \in \mathbb{N}$, otherwise trivial solutions or constant mass setting are, respectively, manifested. Under such settings, a power-law type PDM-deformed harmonic oscillator potential (40) is obtained as

$$
V(x)=\frac{\omega^{2}}{2} \lambda x^{\sigma+2} ; \sigma \neq-2,0 ; \sigma \in \mathbb{N} .
$$

That admits the exact eigenvalues and eigenfunctions of (41) as the solutions of the PDM-Schrödinger equation (37) with $q(x)$ of $(49)$.

\section{A PDM sample case without singularities $m(x)=\left(1+\alpha^{2} x^{2}\right)^{-1}$}

We now start with a PDM function $m(x)$ that has no singularities [21, 34] as

$$
m(x)=\frac{1}{\alpha^{2} x^{2}+1} .
$$

in (11) would imply that

$$
Q(x)=\left[\frac{\ln \left(\alpha x+\sqrt{\alpha^{2} x^{2}+1}\right)+\beta}{\alpha x}\right]^{2} \Longleftrightarrow q(x)=\frac{1}{\alpha}\left[\ln \left(\alpha x+\sqrt{\alpha^{2} x^{2}+1}\right)+\beta\right] .
$$

Consequently a logarithmic-type PDM-deformed harmonic oscillator potential (40) is obtained as

$$
V(x)=\frac{\omega^{2}}{2 \alpha^{2}}\left[\ln \left(\alpha x+\sqrt{\alpha^{2} x^{2}+1}\right)+\beta\right]^{2},
$$

and admits the exact eigenvalues and eigenfunctions of (41) as the solutions of the PDM-Schrödinger equation (37) with $q(x)$ of (52).

\section{E. A PDM sample case with singularities $m(x)=\left(1-\alpha^{2} x^{2}\right)^{-2}$}

A PDM function $m(x)$ with singularities $[21,[34]$

$$
m(x)=\frac{1}{\left(1-\alpha^{2} x^{2}\right)^{2}}
$$

in (11), yields

$$
Q(x)=\frac{1}{4 \alpha^{2} x^{2}}\left[\ln \left(\frac{\alpha x-1}{\alpha x+1}\right)+\beta\right]^{2} \Longleftrightarrow q(x)=\frac{1}{2 \alpha}\left[\ln \left(\frac{\alpha x-1}{\alpha x+1}\right)+\beta\right]
$$

and a logarithmic-type PDM-deformed harmonic oscillator potential (40) is obtained as

$$
V(x)=\frac{\omega^{2}}{8 \alpha^{2}}\left[\ln \left(\frac{\alpha x-1}{\alpha x+1}\right)+\beta\right]^{2},
$$

with its exact eigenvalues and eigenfunctions in (41) as the solutions of the PDM-Schrödinger equation (37) with $q(x)$ of (55). 
F. A PDM in a Morse-type potential $V(x)=A\left(e^{-2 \beta x}-2 e^{-\beta x}\right)$

A Morse-type PDM-deformed harmonic oscillator potential (40)

$$
V(x)=\frac{\lambda \omega^{2}}{2}\left(e^{-2 \beta x}-2 e^{-\beta x}\right),
$$

would suggest that a PDM-deformation $Q(x)$ is given in the form of

$$
Q(x)=\frac{\lambda}{x^{2}}\left(e^{-2 \beta x}-2 e^{-\beta x}\right) \Longleftrightarrow q(x)=\sqrt{\lambda\left(e^{-2 \beta x}-2 e^{-\beta x}\right)},
$$

with a PDM function

$$
m(x)=\lambda \beta^{2} \frac{\left(e^{-\beta x}-1\right)^{2}}{1-2 e^{-\beta x}} .
$$

The corresponding exact eigenvalues and eigenfunctions in (41) as the solutions of the PDM-Schrödinger equation (37) with $q(x)$ of (57).

\section{G. A PDM in Yukawa-type potential $V(x)=-B e^{-\delta x} / x$}

A Yukawa-type PDM-deformed harmonic oscillator potential (40)

$$
V(x)=-\frac{V_{\circ} \omega^{2}}{2}\left(\frac{e^{-\delta x}}{x}\right)
$$

would imply a PDM-deformation function

$$
Q(x)=-V_{\circ} \frac{e^{-\delta x}}{x^{3}} \Longleftrightarrow q(x)=\sqrt{-V_{\circ} \frac{e^{-\delta x}}{x}}
$$

and a PDM function

$$
m(x)=-\frac{V_{\circ}}{4}\left(\frac{\delta x+1}{x^{3}}\right) e^{-\delta x}
$$

The exact eigenvalues and eigenfunctions of which are given in (41) as the solutions of the PDM-Schrödinger equation (37) with $q(x)$ of $(60)$.

\section{CONCLUDING REMARKS}

In this work, we have constructed the PDM creation and annihilation operators for the PDM-deformed harmonic oscillators through two different ways: (i) via von Roos PDM-Hamiltonian (4), and (ii) via a PDM point canonical transformation (25) of the textbook constant mass Hamiltonian analog (21). Using the von Roos Hamiltonian (4), we have shown that the commutation relation (7) (between the PDM creation $\hat{A}^{+}$and annihilation $\hat{A}$ operators for the PDM-deformed harmonic oscillators) offers a strict determination of the PDM kinetic energy operator (18) and suggests a PDM-deformation in the coordinate system (11) (consequently, a PDM-deformation $Q(x)$ in the PDMdeformed harmonic oscillator potential (3)) to imply the PDM Hamiltonian $\hat{H}_{1}$ of (17). On the other hand, the PDM point canonical transformation (25) yields another set of PDM creation $\hat{B}^{+}$and annihilation $\hat{B}$ operators, (26) and (27), for the PDM-deformed harmonic oscillators to result yet another PDM Hamiltonian $\hat{H}_{2}$ of (28). In the process, therefore, two "apparently" different PDM Hamiltonian operators (or equivalently, two different PDM kinetic energy operators) are obtained. In the literature, however, $\hat{H}_{1}$ is known to represent Mustafa and Mazharimousavi's parametric ordering [27] (i.e., $j=\ell=-1 / 4$ and $k=-1 / 2$, obtained through the factorization of the kinetic energy term of (1)). Whereas, $\hat{H}_{2}$ turned out to be correlated with $\hat{H}_{1}$, through (33), but has never been reported as a 
feasible ordering in the literature before. As a result, one PDM Hamiltonian, $\hat{H}_{1}$, is effectively and vividly singled out of the von Roos PDM Hamiltonian (1). The corresponding PDM-Schrödinger equation of which, in general, reads

$$
\left\{\left(\frac{\hat{p}(x)}{\sqrt{2 m(x)}}\right)^{2}+V(q(x))\right\} \phi_{n}(x)=E_{n} \phi_{n}(x),
$$

for any PDM-deformed interaction potential field $V(q(x))$. Where, $\hat{p}(x)=-i\left(\partial_{x}-\frac{1}{4} m^{\prime}(x) / m(x)\right)$.is the PDMmomentum operator very recently reported by Mustafa and Algadhi [10], and

$$
q(x)=\sqrt{Q(x)} x, \sqrt{m(x)}=\sqrt{Q(x)}\left(1+\frac{Q^{\prime}(x)}{2 Q(x)} x\right) .
$$

For the PDM-deformed harmonic oscillator $V(q(x))=\frac{1}{2} \omega^{2} q(x)^{2}=\frac{1}{2} \omega^{2} Q(x) x^{2}$, for example, the PDM creation and annihilation operators are constructed as in (13) and (14), respectively.

Obviously, the PDM-Schrödinger equation (63) as well as the PDM creation and annihilation operators, (13) and (14), look very much like their textbook counterparts for constant mass settings. Yet, they satisfy the textbook commutation relations (20). The correlation

$$
\left(\hat{A} \hat{A}^{+}-\frac{1}{2}\right) \phi(x)=m(x)^{1 / 4}\left(\hat{B} \hat{B}^{+}-\frac{1}{2}\right) \Psi(q(x)),
$$

on the other hand, allows one to build up the energy eigenvalues and eigenfunctions for PDM-deformed harmonic oscillator potential. That is, one may (in the Dirac notations) use

$$
\left.\left.\left.\left.\hat{B} / \Psi_{n}(q(x))\right\rangle=\sqrt{n} / \Psi_{n-1}(q(x))\right\rangle ; \hat{B}^{+} / \Psi_{n}(q(x))\right\rangle=\sqrt{n+1} / \Psi_{n+1}(q(x))\right\rangle ; n=0,1,2, \cdots,
$$

to build up the spectrum through

$$
E_{n}=\omega\left(n+\frac{1}{2}\right)
$$

and

$$
\Psi_{n}(q(x))=m(x)^{-1 / 4} \phi_{n}(x)=\mathcal{N}_{n} \exp \left(-\frac{q(x)^{2}}{2}\right) H_{n}(q(x)) .
$$

In our illustrative examples (section 3), moreover, we have used the PDM-deformation $Q(x)$ to find the corresponding $q(x), m(x)$, and the PDM-deformed harmonic oscillator potential (in the first three examples). Next, we have used the PDM $m(x)$ to obtain the corresponding PDM-deformation $Q(x), q(x)$, and the PDM-deformed harmonic oscillator potential (in the 4th and 5th examples). Then, we have used a Morse-type PDM-deformed harmonic oscillator potential (6th example) and a Yukawa-type PDM-deformed harmonic oscillator potential (7th example) to find the corresponding PDM-deformations $Q(x)$ as well as the corresponding PDM functions $m(x)$.

In the light of our experience through the current methodical proposal, a critical and new observation is unavoidable. The fact that the PDM Hamiltonians $\hat{H}_{1}$ and $\hat{H}_{2}$ are isospectral, (32), and are correlated through (33) immediately suggests that

$$
\hat{H}_{2}=-\frac{1}{2} \frac{1}{\sqrt{m(x)}} \partial_{x} \frac{1}{\sqrt{m(x)}} \partial_{x}+V(q(x)), q(x)=\sqrt{Q(x)} x,
$$

is yet an alternative PDM Hamiltonian which does not belong to the set of von Roos PDM Hamiltonians of (1). Moreover, as long as the eigenvalues and eigenfunctions are to be determined, then $\hat{H}_{2}$ of (68) should, hereinafter, be realized to be yet another equivalent/alternative and viable PDM Hamiltonian. Which, for the PDM-deformed harmonic oscillator discussed above, offers a straightforward construction of the eigenvalues and eigenfunction as well as a new set of PDM creation $\hat{B}^{+}$and annihilation $\hat{B}$ operators, (26) and (27), that can be expressed in terms of the regular constant mass momentum operator $\hat{p}=-i \partial_{x}$, respectively, as

$$
\hat{B}^{+}=-i \frac{\hat{p}}{\sqrt{2 \omega m(x)}}+\sqrt{\frac{\omega}{2}} q(x) \quad, \quad \hat{B}=i \frac{\hat{p}}{\sqrt{2 \omega m(x)}}+\sqrt{\frac{\omega}{2}} q(x) ; \hat{p}=-i \partial_{x} .
$$


Under such new PDM settings, one may now write, in general, the PDM-Schrödinger equation corresponding to $\hat{H}_{2}$ of (68) as

$$
\left\{\left(\frac{\hat{p}}{\sqrt{2 m(x)}}\right)^{2}+V(q(x))\right\} \Psi_{n}(q(x))=E_{n} \Psi_{n}(q(x)),
$$

where, $q(x)$ is given by (64). Finally, all PDM-deformed harmonic oscillators of section 3 , have their exact eigenvalues $E_{n}$ in (66) and eigenfunctions $\Psi_{n}(q(x))$ in (67). This result (70) is, in fact, the most simplistic form of the PDMSchrödinger equation ever reported.

At this point, one should recollect that Cariñena et al. 34] have started with the Killing vector fields for PDM geodesic motion (using a PDM Lagrangian for quasi-free, $V(x)=0$ ) and have quantized the associated Noether momentum (and not the canonical momentum) to come out with a PDM Hamiltonian operator in exact accord with our $\hat{H}_{2}$ of (68) to imply the PDM Schrödinger equation (70). Yet, one should notice that their PDM Noether momentum operator is reported as

$$
\hat{P}=-\frac{i}{\sqrt{2 m(x)}} \partial_{x}=\frac{\hat{p}}{\sqrt{2 m(x)}}
$$

where $\hat{p}=-i \partial_{x}$ is the regular textbook constant mass momentum operator used in (69) and (70).

Finally, the PDM quantum supersymmetric approach with the asymptotic geometrical classical oscillator correspondence by Cruz et al. [21], the factorization approach by Mustafa and Mazharimousavi [27], the construction of the PDM-momentum operator approach by Mustafa and Algadhi [10], and the current PDM creation and annihilation oscillator operators approach, all confirm and emphasize that the PDM-Hamiltonian $\hat{H}_{1}$ of (29) is the only surviving one out of the von Roos PDM Hamiltonians. However, the quantization approach of the PDM Noether momentum by Cariñena et al. [34], and our analysis and discussions in the current methodical proposal suggest that $\hat{H}_{2}$ of (68) is not only correlated with $\hat{H}_{1}$ but also more simplistic user-friendly than $\hat{H}_{1}$ that of (63). It should replace the von Roos PDM-Hamiltonian (1), therefore. 
[1] O. Von Roos, Phys. Rev. B 27 (1983) 7547.

[2] Q. G. Zhu, H. Kroemer, Phys. Rev. B 27 (1983) 3519.

[3] T. Li, K. J. Kuhn, Phys. Rev. B 47 (1993) 12760.

[4] D. J. Ben Daniel, C. B. Duke, Phys. Rev. 152 (1966) 683.

[5] T. Gora, F. Williams, Phys. Rev. 177 (1969) 1179.

[6] P. Ring, P. Schuk, "The Nuclear Many-Body Problem", Springer Science and Business Media, 2004.

[7] R Koc, G Sahinoglu, M Koca, Eur. Phys. J. B 48 (2005) 583.

[8] A. Khlevniuk, V. Tymchyshyn, J. Math. Phys. 59 (2018) 082901.

[9] O. Mustafa, J Phys A: Math. Theor.52 (2019) 148001.

[10] O. Mustafa, Z. Algadhi, Eur. Phys. J. Plus 134 (2019), 228.

[11] O. Mustafa, J. Phys. A; Math. Theor. 48 (2015) 225206.

[12] J. F. Cariñena, M. F. Rañada, M. Santander, M. Senthilvelan, Nonlinearity 17 (2004) 1941.

[13] A. Bhuvaneswari, V. K. Chandrasekar, M. Santhilvelan, M. Lakshmanan, J. Math. Phys. 53 (2012) 073504.

[14] A. K. Tiwari, S. N. Pandey, M. Santhilvelan, M. Lakshmanan, J. Math. Phys. 54 (2013) 053506.

[15] P. M. Mathews, M. Lakshmanan, Quart. Appl. Math. 32 (1974) 215.

[16] B. Bagchi, A. Banerjee, C. Quesne, V. M. Tkachuk, J. Phys. A 38 (2005) 2929.

[17] C. Quesne, J. Math. Phys. 56 (2015) 012903.

[18] O. Mustafa, J. Phys. A: Math. Theor. 46 (2013) 368001.

[19] M. Ranada, M. A. Rodrigues, and M Santander, J. Math. Phys. 51, 042901 (2010).

[20] J. F. Cariñena, F. J. Herranz, M. F. Rañada, J. Math. Phys. 58 (2017) 022701.

[21] S. Cruz y Cruz, J Negro, L. M. Nieto, Phys. Lett. A 369 (2007) 400.

[22] A de Souza Dutra, C A S Almeida, Phys Lett. A 275 (2000) 25.

[23] S. Cruz y Cruz, O Rosas-Ortiz, J Phys A: Math. Theor. 42 (2009) 185205.

[24] O Mustafa, S H Mazharimousavi, Phys. Lett. A 358 (2006) 259.

[25] A D Alhaidari, Phys. Rev. A 66 (2002) 042116.

[26] O Mustafa, S H Mazharimousavi, J. Phys. A: Math. Gen. 39 (2006) 10537.

[27] O Mustafa, S.H. Mazharimousavi, Int. J. Theor. Phys 46 (2007) 1786.

[28] O Mustafa, S H Mazharimousavi, J Phys A: Math. Theor.41 (2008) 244020.

[29] O Mustafa, S H Mazharimousavi, Phys. Lett. A 373 (2009) 325.

[30] O. Mustafa, J Phys A: Math. Theor.43 (2010) 385310.

[31] O. Mustafa, J Phys A: Math. Theor.44 (2011) 355303.

[32] C. Quesne, V. M. Tkachuk, J. Phys. A 37 (2004) 4267.

[33] J. F. Cariñena, M. F. Rañada, M. Santander, Regul. Chaotic Dyn. 10 (2005) 423.

[34] J. F. Cariñena, M. F. Rañada, M. Santander, J. Phys. A: Math. Theor. 50 (2017) 465202.

[35] J. M. Levy-Leblond, Phys. Rev. A 52 (1995) 1845. 